\title{
Libros electrónicos y lectura digital: los escenarios del cambio
}

José Antonio Córdon García

Universidad de Salamanca, España

jcordon@usal.es

Cita sugerida: Córdon García, J. A. (2018). Libros electrónicos y lectura digital: los escenarios del cambio. Palabra Clave (La Plata), 7(2), e044. https://doi.org/10.24215/18539912e044

(c) (i) (s) (2) Esta obra está bajo licencia Creative Commons Atribución-NoComercial-CompartirIgual 4.0 Internacional (c) ${ }_{\text {By }}$ no $\mathrm{sA}$ http://creativecommons.org/licenses/by-nc-sa/4.0/deed.es_AR 


\section{Libros electrónicos y lectura digital: los escenarios del cambio}

\section{Ebooks and digital reading: changing scenarios}

José Antonio Cordón García

Universidad de Salamanca, España

jcordon@usal.es

Nunca han concitado tanta atención los libros y la lectura como ahora, en que los cambios tecnológicos y la posibilidad de una migración similar a la producida en otros géneros, como las obras de referencias y las revistas científicas, preferentemente, han movilizado a una gran parte de los actores implicados en la detracción o defensa de las transformaciones presentes o venideras. Los hechos son una realidad, la edición electrónica ha modificado radicalmente la cadena de valor del libro y de la lectura, aunque el relato vinculado a los mismos cambie según la perspectiva que se adopte.

Una de las singularidades de la situación actual es que las innovaciones revisten un carácter regular y permanente, de tal manera que las iniciativas y propuestas se acumulan en el tiempo dibujando un panorama de posibilidades y oportunidades de una variabilidad imprevisible. $\mathrm{Y}$ en esto, en lo que podíamos cifrar su fragilidad, está también su virtud, pues frente a sistemas precedentes caracterizados por el inmovilismo y cierta pasividad reactiva, nos encontramos ante un modelo basado en la adaptabilidad tecnológica y la flexibilidad empresarial. La concurrencia de los diferentes sistemas de comunicación e información han propiciado, además, que los elementos implicados sean cada vez más numerosos, de tal manera que, frente a anteriores revoluciones de carácter cognitivo, restringidas a un reducido número de personas, la actual se expande al conjunto de una sociedad cada vez más digitalizada.

Es cierto que esta expansión tiene un carácter escasamente homogéneo, y que los libros y la lectura no constituyen sus actividades más relevantes. Pero esta característica es consustancial con una práctica que a lo largo de la historia se ha demostrado como minoritaria y poco intensiva, incluso en sus versiones más prosaicas o superficiales.

Los estudios y encuestas sobre libros y lectura son bastante contradictorios y consecuentemente de escasa fiabilidad. En el informe sobre el sector del libro y la lectura en España de 2017 (Observatorio del libro y la lectura, 2017), se indica que la lectura es la segunda actividad cultural más practicada por los españoles, después de la audición de música, con unos porcentajes que alcanzan al $62 \%$ de la población. Sin embargo, en todas las encuestas sobre prácticas de lectura comprobamos que más de la mitad de la población no ha leído nunca un libro y que los lectores frecuentes constituyen una minoría. Lo mismo ocurre con la lectura digital, que ha experimentado una progresión evidente en los últimos años, pero dentro de un contexto de contención privativo de esta actividad. Los informes coordinados por José Antonio Millán (2002, 2008, 2017) sobre la lectura en España son contundentes. En sus sucesivas ediciones muestran que la lectura progresa globalmente, pero la centrada en los libros cuenta con un núcleo de resistencia fuerte hacia los mismos, en torno al $40 \%$ de la población que, además, en un alto porcentaje argumenta que no lee porque no le interesa (Encuesta de Hábitos y Prácticas Culturales, 2016).

A pesar de esto, si tomamos como referencia el panorama de unos años atrás, podemos comprobar cómo tanto la producción de libros electrónicos como de lectores en digital ha ido en aumento. Este incremento ha estado auspiciado, sin duda, por la ruptura con el campo editorial tradicional, así como con las prácticas de lectura convencionales, expandiendo hacia escenarios nuevos las actividades vinculadas con ambos elementos. Nuevos formatos, nuevos géneros, nuevos comportamientos frente al texto, han 
favorecido la generación de una cultura que, sin romper todavía el cordón umbilical con el entorno impreso, se va adentrando en territorios cada vez más alejados de este. Nos encontramos ante escenarios con un alto grado de experimentalidad, en el que los tiempos de supervivencia de las propuestas son efímeros y a veces imperceptibles, pero en el que se van consolidando tendencias exclusivas del nuevo entorno, con movimientos que beben de la tradición, pero desde una perspectiva completamente renovada, como ocurre con la autopublicación, los audiolibros o los sistemas de recomendación.

¿Qué aportan los nuevos formatos al panorama de la edición y de la lectura?

Karsenti (2017), a partir de diversas investigaciones desarrolladas en la universidad de Montreal, y de la verificación de otras fuentes, establece 25 ventajas de los libros electrónicos para el segmento juvenil de la población. Son interesantes por cuanto reúnen algunas de las características más clarificadoras para identificar los elementos del cambio en la época actual con relación a los inicios del proceso, allá por el año 2007 aproximadamente.

Las características están divididas según ventajas cognitivas, afectivas, sociales, económicas, ecológicas y de portabilidad.

Entre las ventajas cognitivas se cuentan:

- Interactividad: el entorno digital permite la comunicación entre los intervinientes en la elaboración de los contenidos, o con los propios contenidos.

- Acceso: el tiempo y el lugar son dos variables inexistentes en el entorno digital, que permite acceder a los contenidos independientemente del sitio o de la hora.

- Vocabulario: las prestaciones de consulta de palabras y contextualización de las mismas facilita una mejor comprensión de las obras y un mayor enriquecimiento en la lectura.

- Toma de notas: Los sistemas de anotación vinculados con las diversas aplicaciones de lectura son una herramienta muy potente de enriquecimiento de los textos.

- Búsqueda: la posibilidad de recuperar personajes, términos, lugares, favorece el efecto de recordatorio y la asimilación de los contenidos.

- Lectura en voz alta: la función VoiceOver, u otras similares, permiten reducir las dificultades de lectura de aquellos que cuentan con algún tipo de discapacidad.

- Individualización: la adaptabilidad de los textos a las circunstancias particulares de cada lector constituye un poderoso revulsivo para facilitar la legibilidad de los mismos.

- Aprendizaje: la combinación de herramientas de diversa naturaleza en una sola obra favorece los procesos de aprendizaje.

- Actualización: en el entorno digital las actualizaciones adquieren mayor regularidad y fluidez que en el impreso.

- Regularidad de lectura: las estadísticas muestras que los lectores digitales leen más frecuentemente que los lectores de textos impresos.

- Tiempo de lectura: también leen durante más tiempo.

- Organización: las posibilidades de organización y clasificación son mayores en los libros electrónicos.

- Agrupación de prestaciones: en una tableta o un dispositivo móvil se cuenta con una gran diversidad de aplicaciones que pueden emplearse conjuntamente en la lectura.

- Variedad: la cantidad de textos disponibles permite que se pueda elegir entre múltiples opciones.

- Almacenamiento: la capacidad de almacenamiento de los dispositivos de lectura posibilita que se cuente permanente con una biblioteca en permanente expansión.

Entre las ventajas afectivas:

- Interés: la familiaridad con el entorno digital atrae a los jóvenes hacia cualquier producto derivado del mismo. 
- Multimedia: la integración de contenidos de sonido, imagen y texto favorece el efecto de gamificación y de interacción con los mismos.

- Confortabilidad: la experiencia de lectura digital es agradable y atractiva para los lectores más jóvenes.

- Adaptación: la personalización de dispositivos y de aplicaciones permite que cada lector articule su entorno de lectura.

Entre las ventajas sociales:

- Socialización: el desarrollo de la lectura social permite que se pueda compartir fácilmente aquello que interesa entre lo que se lee.

- Intercambio: el intercambio de lecturas de interés a través de diferentes medios forma parte consustancial de los libros electrónicos

- Colaboración: todas las herramientas de socialización facilitan igualmente la colaboración.

A estas habría que añadirles las económicas, en tanto que los libros electrónicos representan ahorros considerables respecto a los impresos, las ecológicas por cuanto el impacto es menos negativo sobre el medioambiente, y la portabilidad en tanto que se pueden llevar numerosos libros sin los inconvenientes del volumen y el peso.

Estas ventajas indudables no determinan un incremento de la lectura perceptible, ni la migración previsible del impreso a digital vaticinada hace años, y que ya había verificado en otras modalidades como la de las revistas y las obras de referencia. La secuencia de los libros en este proceso de cambio es bastante más lenta de lo anticipado y con fuertes núcleos de resistencia en segmentos de la población que, presumiblemente, habrían de ser más proclives hacia los nuevos medios.

Las cifras sobre el mercado de libros electrónicos de 2015 a 2017 en los principales mercados de los mismos, Estados Unidos e Inglaterra, muestran un declive en su crecimiento y una tendencia al estancamiento, en contraste con lo que ocurre con otras industrias culturales como el cine o la música y, por supuesto, en relación con el libro impreso. Un estudio publicado por Publishers Weekly (Milliot, 2016) abunda en lo mismo. Además de factores de carácter contextual, se apunta como explicación al síndrome de "fatiga digital" que tiene que ver con la usabilidad de los dispositivos, con el cansancio atribuible a la conectividad forzada, y con el uso excesivo de pantallas. En una encuesta desarrollada por el Grupo Codex, en Estados Unidos, el 25\% de los encuestados compradores de libros electrónicos confiesan la necesidad de distanciarse de sus dispositivos móviles, y un 19\% de los entrevistados confiesan que leen menos libros electrónicos en la actualidad que cuando comenzaron a emplear esos formatos, manifestando una preferencia por la vuelta a los formatos impresos. Lo curioso es que esta voluntad de retorno es inversamente proporcional con la edad, es decir que son los más jóvenes, los que pasan más tiempo entre pantallas, los que manifestarían una mayor inclinación a retomar las prácticas de lectura impresa.

Otro estudio desarrollado por investigadores de tres universidades australianas (Merga, Roni y Cowan, 2017), muestra cómo los niños que acceden regularmente a dispositivos electrónicos (sin diferenciar entre tabletas, teléfonos inteligentes y dispositivos de tinta electrónica) apenas los usan para leer, aunque sean lectores regulares en papel, que prefieren al digital. Un dato interesante del estudio es que se da, igualmente, una relación inversamente proporcional entre uso de los dispositivos y niveles de lectura.

Zielinski (2013), advierte sobre el peligro de la sobreexposición al entorno digital, del que sin duda una consecuencia puede ser esta, pero esto forma parte del relato de los hechos a los aludíamos al principio, y no de los hechos, que admiten explicaciones menos lineales.

Es cierto que la fatiga digital como teoría puede cautivar por su simplicidad, pero la perspectiva desde la que se aborda es excesivamente corta como para articular una tendencia verdaderamente consistente, amparada además por unas estadísticas de escaso recorrido todavía. Sin embargo, constituye un excelente ejemplo para analizar cómo funciona, en el nivel más ideologizado, el análisis de las tendencias relativas a los 
contenidos digitales. En algunos de los estudios que llegan a conclusiones similares no se tiene en cuenta ni los tipos de obras que se leen ni el tipo de dispositivo en el que efectúa la lectura, más allá de identificar que se trata de contenidos digitales. La literatura científica muestra cómo los niveles de compresión lectora están directamente relacionados con el tipo de pantalla que se emplea, sus dimensiones y prestaciones. Un estudio desarrollado en la universidad de Alberta en 2010, y replicado en 2016 (Meyer, 2016), demuestra que los niveles de comprensión y la velocidad de lectura de contenidos complejos están determinados por el tipo de pantalla empleado para ello. Pero también con el tipo de aplicaciones que se emplean. Gómez Díaz, et al. (2016), demostraron que los parámetros en los que se mueven dispositivos y programas entrañan cientos de posibilidades de combinación con ratios mayores o menores de calidad y usabilidad en función de las mismas.

El problema no es solo de cifras, sino del valor que atribuimos a las mismas y los conceptos sobre los que descansan. Un estudio desarrollado entre varios miles de niños y adolescentes ingleses (Topping, 2017), mostraba cómo en una gran cantidad de casos los lectores saltan páginas e incluso capítulos enteros en sus lecturas. A pesar de que sea una práctica recomendada por el inefable Daniel Pennac, ¿se han de computar como libros leídos los pertenecientes a este grupo? La lectura digital iha de restringirse a los libros o ha de contemplar otras realidades distintas de los mismos, habida cuenta de la emergencia de nuevos géneros y nuevas prácticas? Las estadísticas de obras autopublicadas ¿se ha de integrar en la de los libros computados por en la producción editorial de cada país? La lectura de libros ¿incluye también la audición de los mismos? ¿Dónde se ubican las obras transmedia en la categorización estadística?

Son muchas las interrogantes que se pueden plantear en este nuevo entorno, a alguna de ellas se responde de manera solvente por parte de los autores de este monográfico que plantean diversos temas relacionados con la lectura digital, en términos generales (Isabel Morales) o en un contexto determinado, la universidad (Olivia Jarvio); con las aplicaciones de lectura en entornos sociales (Taisa Dantas), o en los espacios de la biblioteca (Raquel Gómez y Araceli García); con los sistemas de recomendación (Luis Miguel Cencerrado, Sofia Pelosi y Elisa Yuste); con la autopublicación (Almudena Mangas, Javier Merchán y Alberto Ramos), con los conceptos de Canon y Corpus digital (Adrian Vila).

Un elenco de intervenciones que tocan muchos de los puntos sensibles de estas nuevas prácticas que se vienen articulando desde hace por lo menos 10 años, cuando de forma incipiente, y a la luz de la aparición del Kindle de Amazon, empieza a gestarse un ecosistema formado por un entramado de integración vertical en el que creación de contenidos, plataformas de distribución y dispositivos de lectura propician por primera vez un entorno favorable a la lectura digital. Desde entonces, año 2007, no se ha producido la inexorable irrupción del libro electrónico con la potencia devastadora de una revolución que barriera con todo lo precedente. Antes bien, el mercado demuestra que este formato está sujeto a un crecimiento regular pero constante, con algunos retrocesos, de carácter coyuntural, pero bien posicionado en el sector editorial. La revolución se ha producido en las prácticas de lectura que han experimentado cambios radicales en relación con las desarrolladas en el referente impreso.

La lectura se ha convertido en social, como se puede percibir contemplando el contingente de tweets, post o publicaciones que generan los cientos de hashtag relacionados con libros y autores, o visitando las numerosas redes sociales especializadas que han surgido en los últimos años, como Goodreads, Library Think, Entre lectores, etc., que cuentan con miles de participantes. Para los integrantes de la cadena del libro esto implica que la prescripción y la influencia se ha transferido en parte a los lectores, y que de una prescripción descendente (críticos, medios, librerías, bibliotecas) se ha producido el desplazamiento a una ascendente, en la que los lectores son cada vez más sensibles a los comentarios, notas y discusiones que aparecen en estas plataformas sociales. Blogueros, youtubers y otros actores de este micro marketing digital se han convertido en referentes para cientos de miles de usuarios que siguen con fidelidad sus recomendaciones, más efectivas desde el punto de vista de la difusión de una obra que la tradicional publicidad, dada la posibilidad de alcanzar a comunidades de intereses más especializadas. Gracias a ello se establecen relaciones basadas en la confianza, la autenticidad y la proyección, generando por lo tanto niveles de compromiso reforzados por 
los algoritmos de Facebook, Instagram o Youtube. Las comunidades de lectores, los fans fictions, los grupos vinculados a clubes de lectura digital crecen y desarrollan prácticas solo posibles gracias a las prestaciones tanto de las aplicaciones de lectura como de las redes vinculadas a las mismas. Las aplicaciones de lectura se comportan de manera diferente, según el entorno (Ios, Android), o el dispositivo empleado para su ejecución (tablet, phablet, móvil, ordenador, dispositivo dedicado). Su conocimiento es fundamental para vislumbrar sus posibilidades y limitaciones. A ello está dedicado el artículo de Taisa Dantas, en el que se puede leer un sistemático y riguroso análisis de algunas de las app más empleadas en diferentes plataformas. Pero también se hace imprescindible analizar estas aplicaciones en un entorno que se verá afectado directamente por ellas como es el de la biblioteca, en trance de completa transformación debido a los cambios operados en la lectura en los últimos años. Las autoras Raquel Díaz y Araceli García abogan por la necesaria convivencia entre lo analógico y lo digital dentro de este contexto de cambio, plasmando las particularidades de la lectura infantil y sus necesidades. En esta línea la recomendación de contenidos digitales para niños y jóvenes representa uno de los retos de los actuales sistemas de prescripción. En unas edades donde la economía de la atención alcanza un carácter crítico, la labor de orientación en el consumo de contenidos es esencial, con objeto de facilitar la tarea de todos los actores que pivotan en torno a este sector de la población. Las funciones del recomendador, la naturaleza de sus tareas y la finalidad de las mismas es objeto de una atención esclarecedora en la contribución de Cencerrado, Pelosi y Yuste. Caroline Myrberg (2017) se planteaba en un artículo reciente por qué hay tantos estudiantes a los que no les gusta leer libros electrónicos y prefieren el papel. La respuesta, como apunta la autora, tiene que ver con factores implícitos en el propio formato, la usabilidad de los dispositivos, las expectativas del lector y otras circunstancias que, en el ámbito universitario, analiza también Olivia Jarvio, explorando las posibilidades de las nuevas prácticas de lectura en un contexto en cierto modo especializado, centrándose en una práctica singular como es la de la lectura no utilitaria, un término sumamente sugerente por las implicaciones conceptuales y metodológicas que implica. Pero la respuesta radica también en otros supuestos vinculados con las culturas imperantes, con las pugnas por la conquista de los espacios de creación y de interpretación, por la inserción de prácticas que revelan los graves contrastes de mentalidades discursivas existentes en el relato de lo literario. Una interesante exploración hermenéutica es que la desarrolla Isabel Morales para desbrozar los inextricables senderos de lo digital desde el punto de vista de la tradición literaria. Y si de tradición se trata, nada más representativo de la misma que las nociones de Canon y de Corpus, un fenómeno transversal que atraviesa la historia del libro y de la lectura desde hace siglos. La selección de las mejores obras, la evaluación, la crítica, la interpretación, la glosa y la prescripción se asocian a un movimiento que implícitamente o explícitamente subyace en todo recorrido académico que se haga del mundo editorial. Bloom lo puso de moda hace años, pero referido al entorno de lo publicado en algún tipo de soporte físico. Adrián Vila amplía el campo de estudio al ámbito digital, con todos los problemas de marco teórico y metodológico que ello comporta, y al contexto latinoamericano y caribeño, ilustrando los problemas de construcción de un corpus a partir de fuentes muy diversas, en las que no se elude la heterodoxia heurística. Nada más heterodoxo, y contrario al canon, que la autopublicación. Basada en la desregularización del sistema editorial, en la supresión de las intermediaciones, el fenómeno, no nuevo como ponen de manifiesto Almudena Mangas, Javier Merchán y Alberto Ramos, ha encontrado un particular encaje en las plataformas dedicadas a ello en la red. La proliferación de obras autopublicadas en todo el mundo, con cifras que se cuentan por cientos de miles de títulos, muestran una realidad disruptiva y novedosa, vinculada con el afán por escribir y darle visibilidad a lo escrito, pero en el polo opuesto de la calidad, donde se articula el canon. Si la excelencia constituye la seña de identidad de este, la mediocridad, cuando no la precariedad formal y compositiva, gramatical y conceptual, representa las circunstancias de aquel afán mencionado. La literatura científica sobre el mismo y sobre su aprovechamiento en bibliotecas es cada vez más significativa, y a ella se dedica el interesante análisis de los autores arriba citados.

Tenemos ante nosotros un volumen rico y versátil en temas y propuestas, tratados por algunos de los mejores especialistas del momento en libros electrónicos y lectura digital. Si el debate es el alma de la vida 
académica, en este monográfico se podrán encontrar argumentos para alimentar muchos de ellos, para proseguir las conversaciones aquí iniciadas, para continuar con unas reflexiones inmersas en el corazón de la cultura, de la tradición y de la innovación.

\section{ReFERENCIAS}

Encuesta de Hábitos y Prácticas Culturales 2014-2015. Ministerio de Cultura, 2016.

Gómez Díaz, R., García Rodríguez, A., Cordón García, J. A. y Alonso Arévalo, J. (2016). Leyendo entre pantallas. Gijón: Trea.

Karsenti, M. A. (2017). 25 avantages du livre numerique. Université de Montreal. Recuperado de https://fondationlitterairefleurdelys.wordpress.com/2015/01/29/25-principaux-avantages-du-livre-numeri que-par-thierry-karsenti-m-a-m-ed-ph-d/

Merga, M. K, Roni, S. M. y Cowan, E. (2017). The influence of access to eReaders, computers and mobile phones on children's book reading frequency. Computer and Education, 109, 187-196.

Meyer, K (2016). Reading Content on Mobile Devices. Recuperado de https://www.nngroup.com/articles/mobilecontent/

Millán, J. A. (coord.) (2002, 2008, 2017). La lectura en España: Informe. Federación de Gremios de Editores.

Milliot, J. (2016). As E-book Sales Decline, Digital Fatigue Grows. Publishers Wekly. Recuperado de https://www.publishersweekly.com/pw/by-topic/digital/retailing/article/70696-as-e-book-sales-decline-di gital-fatigue-grows.html

Myrberg, C. (2017). Why doesn't everyone love reading e-books? Insights, 30(3), 115-125. http://doi.org/10.1629/ uksg.386

Observatorio del Libro y de la Lectura (2017). El sector del libro en España. Recuperado de https://www.mecd.gob.e s/dam/jcr:b944f319-8df8-4e2c-aeac-e2043013954c/InfografiaSectorLibro-Octubre\%202017.pdf

Topping, K. (2017). What Kids Are Reading report 2017. Recuperado de http://whatkidsarereading.co.uk/downlo $\mathrm{ad} /$ download-the-full-report/

Zielinski, S. (2013). After the Media: News from the Slow-Fading Twentieth Century. Univocal Publishing. 